\title{
On a parabolic-elliptic chemotactic model with coupled boundary conditions ${ }^{1}$
}

\author{
Manuel Delgado $^{1}$, Cristian Morales-Rodrigo ${ }^{1}$, Antonio Suárez ${ }^{1}$ and J. \\ IGNACIO TELLO ${ }^{2}$
}

1. Dpto. de Ecuaciones Diferenciales y Análisis Numérico

Fac. de Matemáticas, Univ. de Sevilla

Calle Tarfia s/n, 41012-Sevilla, Spain

2. Departamento de Matemática Aplicada,

Escuela de Informática, Universidad Politécnica de Madrid, Carretera de Valencia Km 7. Campus Sur. 28031-Madrid, Spain

E-mail addresses: madelgado@us.es, cristianm@us.es, suarez@us.es, jtello@eui.upm.es

\begin{abstract}
This paper deals with a nonlinear system of parabolic-elliptic type with a logistic source term and coupled boundary conditions related to pattern formation. We prove the existence of a unique positive global in time classical solution. We analyze also the stationary problem associated. Moreover it is proved, under the assumption of sufficiently strong logistic dumping, that there is only one nonzero homogeneous equilibrium, and all the solutions to the non-stationary tend to this steady-state for large times.
\end{abstract}

AMS Classification. 35K45, 35K57, 92C17.

Keywords. Chemotaxis, Global existence, Asymptotic behavior, Coexistence states.

\section{Introduction}

In many areas of research, from the biology of the embryonic development to the tumoral growth, the models of the cell movement play a fundamental role. Broadly speaking, the continuous models fall in two main classes: the mechanochemical models and the chemotactic models. In the former, cells exert a traction on the extracellular matrix (ECM), which carries out a key role; in the latter, cells secrete a chemical substance, which attracts or repels, and move towards or away from the gradient of this chemical. There exists a large number of examples where both types of models are applied to describe different biological phenomena. Numerical simulations are used to compare the experimental data with the mathematical results and to justify these mathematical models (see, [7], [12]). In any case, the study of these systems have interest by itself (see, for instance, [13]).

On the other hand, we must not forget the influence of the boundary conditions on the behavior of the solutions. Recently, nonlinear boundary conditions have been incorporated

\footnotetext{
${ }^{1} \mathrm{MD}$ and AS have been supported by the Spanish Ministry of Science and Technology under Grant MTM2006-07932 and JIT by Spanish Ministry of Sciences and Technology under grant MTM2009-13655.
} 
permitting a more accurate consideration of several situations (see, for instance, [6] and references therein).

In [10], a generalized chemotactic model is considered whose origin was the spatial pattern formation in embryology. It includes a generalized boundary condition which permits to cover a number of them varying the control parameters. We think it is not difficult to apply these models in other fields, for example, in angiogenesis, the cell movement linked to the tumoral growth where the endothelial cells (ECM) move following the gradient of TAF, the chemicals secreted by the tumor cells; for this reason, we think it is interesting to study it. The nondimensional model is

$$
\begin{cases}u_{t}=D \Delta u-\chi \nabla \cdot(u \nabla v)+\mu s u(1-u) & \text { in } \Omega \times(0, T), \\ v_{t}=\Delta v-s v+s \frac{u}{\gamma+u} & \text { in } \Omega \times(0, T), \\ \rho_{1}\left(D \frac{\partial u}{\partial n}-\chi u \frac{\partial v}{\partial n}\right)=\left(1-\rho_{1}\right)\left(\rho_{2}-u\right), \rho_{3} \frac{\partial v}{\partial n}=\left(1-\rho_{3}\right)\left(\frac{\rho_{2}}{1+\gamma}-v\right) & \text { on } \partial \Omega \times(0, T), \\ u(x, 0)=u_{0}(x) & \text { in } \Omega,\end{cases}
$$

where $\Omega \subset \mathbb{R}^{d}$ is a bounded domain with regular boundary, $u$ is the cell density, $v$ is the concentration of chemoattractant, $D>0$ is the diffusion rate, $\chi>0$ is the chemotactic coefficient of the motile cells, $\mu$ is the linear growth rate of the cell population, $\gamma$ is a constant governing the rate of chemoattractant production and degradation and $s$ is a parameter which controls spatial and temporal scale. In [10], it is presented numerical solutions of this model in one spatial dimension and is discussed the behavior of the model in two dimensions. The authors consider also the possibility to give different values to the parameters $\rho_{1}, \rho_{2}, \rho_{3}$ in different parts of the boundary.

During this work, we consider the following system of equations

$$
\begin{cases}u_{t}=\Delta u-\chi \nabla \cdot(u \nabla v)+\mu u(1-u) & \text { in } \Omega \times(0, T), \\ 0=\Delta v-v+\frac{u}{1+u} & \text { in } \Omega \times(0, T), \\ \frac{\partial u}{\partial n}-\chi u \frac{\partial v}{\partial n}=r(\theta-u), \quad \frac{\partial v}{\partial n}=r^{\prime}\left(\frac{\theta}{2}-v\right) & \text { on } \partial \Omega \times(0, T), \\ u(x, 0)=u_{0}(x) & \text { in } \Omega,\end{cases}
$$

where $\mu, r, r^{\prime}, \theta, \chi$ denote non-negative constants. As we can see, it is the model resulting from (1) for $D=s=\gamma=1, \rho_{2}=\theta, r=\frac{1-\rho_{1}}{\rho_{1}}, r^{\prime}=\frac{1-\rho_{3}}{\rho_{3}}$ and supposing that the temporal scale of chemical diffusion is much larger than the scale of diffusion of cells and consequently we can take $v_{t}=0$. We will suppose that $\theta, r$ and $r^{\prime}$ are nonnegative constants on $\partial \Omega$. Since we are interested only in non-negative solutions we assume that $u_{0}(x) \geq 0$ in $\Omega$.

Our purpose is the theoretical study of (2) and its associated stationary problem associated. Our main results can be summarized as follows:

a) The parabolic problem has existence and uniqueness of global solution $\forall r, r^{\prime}, \theta, \mu \geq$ 0 , for suitable initial data.

b) With respect to the elliptic problem, we can summarize the situation as follows:

(a) If $\theta>0$, 
- there exists at least a coexistence state $\forall r, r^{\prime}, \mu \geq 0$,

- there exists the trivial solution if, and only if, $r=r^{\prime}=0$; which is unstable for $\mu>0$,

- there exists one semitrivial solution $(0, v)$ if $r=0, r^{\prime}>0$; which is unstable for $\mu>0$.

In particular, if $\theta=1$, the system has the homogeneous solution $(u, v)=$ $(1,1 / 2)$. Then, we prove that

- if $r, r^{\prime}>0$, then $(1,1 / 2)$ is globally stable when $\mu \geq 0$,

- if $r^{\prime}=0, r \geq 0$, then $(1,1 / 2)$ is globally stable when $\mu$ is big enough.

(b) If $\theta=0$, there exists the trivial solution and there is no semitrivial solution $(0, V)$. We can find three curves, $\mu=h_{1}(r), \mu=h_{2}(r), \mu=h_{3}(r)$, being $h_{1}(r) \geq h_{2}(r) \geq h_{3}(r)$, such that

- if $\mu>h_{1}(r)$, then there exists a coexistence state,

- if $\mu<h_{3}(r)$, then there exists no coexistence state,

- if $\mu<h_{2}(r)$, then the trivial solution is stable.

It is worthy to be remarked that we use a fixed point argument to study the evolutive problem due to the fact that our system has one parabolic equation and one elliptic equation and the general theory for parabolic equations (which is used, for example, in [8]) has to be applied in a non-local framework, less constructive than the method we follow. On the other hand, the method of bifurcation to study the stationary problem (see also [8]) needs the knowledge of some nonnegative solution of the system to begin the branch of positive solutions; this solution is usually one of the semitrivial solutions, but in our case the system does not admit any semitrivial solution when $r, r^{\prime}$, and $\theta$ are positive and, for this reason, we use a decoupling method.

The paper is organized as follows. In Sections 2 and 3, we study the existence and uniqueness of the global solution for the parabolic problem (2). This problem has only one possible constant coexistence state for $\theta=1,(u, v)=\left(1, \frac{1}{2}\right)$; we study in this case the asymptotic behavior of the solutions of (2) in Section 4. In Section 5, we consider the steady problem associated to our system, by a decoupling method and a sub-supersolution method for nonlocal problems. We will obtain some results of existence of solutions and some results of stability of the semitrivial solutions when they exist.

\section{Preliminaries}

First we observe that

$$
\chi \nabla \cdot(u \nabla v)=\chi(\nabla u \cdot \nabla v+u \Delta v)=\chi\left(\nabla u \cdot \nabla v+u\left(v-\frac{u}{1+u}\right)\right) .
$$

So, we may rewrite the system (2) as follows

$$
\begin{cases}u_{t}=\Delta u-\chi \nabla u \cdot \nabla v+\chi u\left(\frac{u}{1+u}-v\right)+\mu u(1-u) & \text { in } \Omega \times(0, T), \\ 0=\Delta v-v+\frac{u}{1+u} & \text { in } \Omega \times(0, T), \\ \frac{\partial u}{\partial n}=\chi u r^{\prime}\left(\frac{\theta}{2}-v\right)+r(\theta-u), & \frac{\partial v}{\partial n}=r^{\prime}\left(\frac{\theta}{2}-v\right) \\ u(x, 0)=u_{0}(x) & \text { on } \partial \Omega \times(0, T), \\ \text { in } \Omega .\end{cases}
$$


Next, in order to avoid the singularity of $\frac{u}{u+1}$ we define the penalized function

$$
h(u):=\frac{u_{+}}{u_{+}+1}
$$

and we introduce the system

$$
\begin{cases}u_{t}=\Delta u-\chi \nabla u \cdot \nabla v+\chi u\left(h(u)-v+\frac{\mu}{\chi}(1-u)\right) & \text { in } \Omega \times(0, T), \\ 0=\Delta v-v+h(u) & \text { in } \Omega \times(0, T), \\ \frac{\partial u}{\partial n}=\chi u r^{\prime}\left(\frac{\theta}{2}-v\right)+r(\theta-u), \quad \frac{\partial v}{\partial n}=r^{\prime}\left(\frac{\theta}{2}-v\right) & \text { on } \partial \Omega \times(0, T), \\ u(x, 0)=u_{0}(x) & \text { in } \Omega .\end{cases}
$$

Observe that we may consider the equation for $u$ as a linear equation

$$
\begin{gathered}
U_{t}-\Delta U=-\chi a(x, t) \cdot \nabla U+\chi b(x, t) U \text { in } \Omega, \\
\frac{\partial U}{\partial n}=\chi r^{\prime} c(x, t) U+r(\theta-U) \text { on } \partial \Omega .
\end{gathered}
$$

where

$$
\begin{aligned}
a(x, t) & :=\nabla v, \\
b(x, t) & :=h(u)-v+\frac{\mu}{\chi}(1-u), \\
c(x, t) & :=\left(\frac{\theta}{2}-v\right) .
\end{aligned}
$$

Thus, if $b(x, t) \in L^{\infty}\left(\Omega \times\left(0, T_{\max }\right)\right)$, then, by the maximum principle, $U(x, t)=u(x, t) \geq 0$ in $\Omega \times[0, T)$ and a solution to (4) is a solution to (3) and viceversa. Now, having in mind that for positive solutions the systems (3) and (4) are equivalent if $u, v \in L^{\infty}\left(\Omega \times\left(0, T_{\max }\right)\right)$, we will show the local existence theorem for (4). Previously we prove the following result where the notation is taken from [2], Sections 6 and 7 :

Lemma 2.1 Let $p>1,1<\beta<2 \alpha<1+\frac{1}{p}$ and $\left(W_{\mathcal{B}}^{2 \alpha-2, p}, A_{\alpha-1}\right)$ an element of the interpolation-extrapolation scale generated by $A_{0}:=-\Delta+I$ and the real interpolation functor, then

$$
\left\|e^{-t A_{\alpha-1}} u\right\|_{W^{\beta, p}} \leq C e^{-\nu t} t^{-\kappa}\|u\|_{W_{\mathcal{B}}^{2 \alpha-2, p}}
$$

with $\kappa(\beta) \in(0,1)$ and $\nu \in(0,1)$.

Proof. By Theorem 7.2 of $[2]$, we have $\left(W_{\mathcal{B}}^{2 \alpha, p}, W_{\mathcal{B}}^{2 \alpha-2, p}\right)_{\kappa, p}=W_{\mathcal{B}}^{\beta, p}$, therefore, using this reference, we know that there exists $\kappa \in(0,1)$ such that

$$
\left\|e^{-t A_{\alpha-1}} u\right\|_{W_{\mathcal{B}}^{\beta, p}} \leq\left\|e^{-t A_{\alpha-1}} u\right\|_{W_{\mathcal{B}}^{2 \alpha, p}}^{\kappa}\left\|e^{-t A_{\alpha-1}} u\right\|_{W_{\mathcal{B}}^{2 \alpha-2, p}}^{1-\kappa} .
$$

Next, we apply [2, Theorem 8.5$]$ together with $[2,(3.1)]$ and we get

$$
\left\|e^{-t A_{\alpha-1}} u\right\|_{W_{\mathcal{B}}^{\beta, p}} \leq\left\|\left(I+A_{\alpha-1}\right) e^{-t A_{\alpha-1}} u\right\|_{W_{\mathcal{B}}^{2 \alpha-2, p}}^{\kappa}\left\|e^{-t A_{\alpha-1}} u\right\|_{W_{\mathcal{B}}^{2 \alpha-2, p}}^{1-\kappa}
$$


Taking into account that $I+A_{\alpha-1}$ and $A_{\alpha-1}$ are sectorial operators with $\operatorname{Re} \sigma\left(I+A_{\alpha-1}\right)=$ $1+\operatorname{Re} \sigma\left(A_{\alpha-1}\right)=2$ then we invoke [9, Theorem 1.3.4]. Thus, there exist $\nu \in(0,1)$, $\alpha \in(0,2)$ such that

$$
\begin{aligned}
\left\|e^{-t A_{\alpha-1}} u\right\|_{W_{\mathcal{B}}^{\beta, p}} & \leq\left\|\left(I+A_{\alpha-1}\right) e^{-t\left(I+A_{\alpha-1}\right)} e^{t I} u\right\|_{W_{\mathcal{B}}^{2 \alpha-2, p}}^{\kappa}\left\|e^{-t A_{\alpha-1}} u\right\|_{W_{\mathcal{B}}^{2 \alpha-2, p}}^{1-\kappa} \\
& =e^{\kappa t}\left\|\left(I+A_{\alpha-1}\right) e^{-t\left(I+A_{\alpha-1}\right)} u\right\|_{W_{\mathcal{B}}^{2 \alpha-2, p}}^{\kappa}\left\|e^{-t A_{\alpha-1}} u\right\|_{W_{\mathcal{B}}^{2 \alpha-2, p}}^{1-\kappa} \\
& \leq e^{(\kappa(1-\alpha)-\nu(1-\kappa)) t} t^{-\kappa}\|u\|_{W_{\mathcal{B}}^{2 \alpha-2, p} .}
\end{aligned}
$$

Finally, we pick $1-\alpha=-\nu$ and use the fact that $W_{\mathcal{B}}^{\beta, p}=W^{\beta, p}$ to conclude the proof.

Lemma 2.2 Let $1<\beta<2 \alpha<1+\frac{1}{p}$ then there exists $\kappa<1$ such that $X_{\kappa} \hookrightarrow W^{\beta, p}$, where $X_{\kappa}:=D\left(\left(I+A_{\alpha-1}\right)^{\kappa}\right)$

Proof. Arguing in the same manner as we did in the previous lemma we have that there exists $\theta<1$ such that

$$
\|u\|_{W^{\beta, p}} \leq C\left\|\left(I+A_{\alpha-1}\right) u\right\|_{W^{2 \alpha-2, p}}^{\kappa}\|u\|_{W^{2 \alpha-2, p}}^{1-\kappa} .
$$

Finally the lemma can be concluded with the use of [9, pg. 28, Exer. 11].

\section{Global existence in time}

Theorem 3.1 Let $p>d$ and consider the initial data $u_{0} \in W^{1, p}(\Omega)$ with $u_{0} \geq 0$. Then there exists $\tau\left(\left\|u_{0}\right\|_{W^{1, p}}\right)$ such that the system (4) has a unique positive local in time solution

$$
(u, v) \in\left(\mathcal{C}\left([0, \tau] ; W^{1, p}(\Omega)\right) \cap \mathcal{C}^{1}\left((0, \tau) ; \mathcal{C}^{2+\alpha}(\bar{\Omega})\right)\right)^{2},
$$

and $u(x, t), v(x, t) \geq 0$ for $(x, t) \in \bar{\Omega} \times[0, \tau]$. Moreover, the solution depends continuously on the initial data, i.e. if $\mathbf{u}\left(u_{0}\right)$ and $\mathbf{u}\left(\bar{u}_{0}\right)$ denote the solutions to (4) with inial data $u_{0}$ and $\bar{u}_{0}$ respectively then

$$
\left\|\mathbf{u}\left(u_{0}\right)-\mathbf{u}\left(\bar{u}_{0}\right)\right\|_{\left(\mathcal{C}\left([0, \tau] ; W^{1, p}\right)\right)^{2}} \leq C\left\|u_{0}-\bar{u}_{0}\right\|_{W^{1, p}} .
$$

Proof. The proof of the Theorem is based on a standard fixed point argument. Let

$$
X_{T}:=\mathcal{C}\left([0, T] ; W^{1, p}(\Omega)\right) .
$$

For each $f \in X_{T}$ we consider the operator

$$
\begin{aligned}
S: X_{T} & \rightarrow \mathcal{C}\left([0, T] ; W^{2, p}(\Omega)\right) \\
f & \mapsto S(f)=v
\end{aligned}
$$

where $v$ is the unique solution to

$$
\left\{\begin{array}{lc}
-\Delta v+v=h(f) & \text { in } \Omega \times(0, T), \\
\frac{\partial v}{\partial n}=r^{\prime}\left(\frac{\theta}{2}-v\right) & \text { on } \partial \Omega \times(0, T) .
\end{array}\right.
$$


Moreover for each $t \in[0, T]$, thanks to [1], the following estimate is satisfied

$$
\|v(t)\|_{W^{2, p}} \leq C(t)\left(\|h(f)\|_{p}+\left\|\theta / 2 r^{\prime}\right\|_{W^{1-1 / p, p}(\partial \Omega)}\right) .
$$

Next, we consider the operator

$$
\begin{aligned}
H: X_{T} & \rightarrow X_{T} \\
f & \mapsto H(f)=u,
\end{aligned}
$$

where $u$ is the unique solution to the linear parabolic problem

$$
\begin{cases}u_{t}-\Delta u=-\chi \nabla f \cdot \nabla v+\chi f(h(f)-v)+\mu f(1-f) & \text { in } \Omega \times(0, T), \\ \frac{\partial u}{\partial n}=\chi f r^{\prime}\left(\frac{\theta}{2}-v\right)+r(\theta-f) & \text { on } \partial \Omega \times(0, T) .\end{cases}
$$

Let $2 \alpha \in\left(1,1+\frac{1}{p}\right)$. Thanks to the generalized variations of constants formula, see [2] pg. 63 , we can rewrite (7) in the following manner

$$
u(t)=e^{-t A_{\alpha-1}} u_{0}+\int_{0}^{t} e^{-(t-\tau) A_{\alpha-1}}\left(F(f, v)+A_{\alpha-1} \mathcal{B}^{c} \gamma g(f, v)\right) d \tau,
$$

where $\gamma: W^{1, p}(\Omega) \rightarrow W^{1-1 / p, p}(\partial \Omega)$ denotes the trace operator and

$$
\begin{aligned}
& F(f, v):=-\chi \nabla f \cdot \nabla v+\chi f(h(f)-v)+\mu f(1-f), \\
& g(f, v):=\chi f r^{\prime}\left(\frac{\theta}{2}-v\right)+r(\theta-f) .
\end{aligned}
$$

Let us point out that, since $A_{\alpha-1} \mathcal{B}^{c} \in \mathcal{L}\left(W^{2 \alpha-1-1 / p, p}(\partial \Omega), W_{\mathcal{B}}^{2 \alpha-2, p}\right)$ together with the embedding $W^{1-1 / p, p}(\partial \Omega) \hookrightarrow W^{2 \alpha-1-1 / p, p}(\partial \Omega)$, then we can assert that $A_{\alpha-1} \mathcal{B}^{c} \gamma$ is well defined for $g(f, v) \in W^{1, p}(\Omega)$. Next, we define the closed set

$$
B_{R}^{T}:=\left\{f \in \mathcal{C}\left([0, T] ; W^{1, p}(\Omega)\right):\|f\|_{X_{T}} \leq R\right\} .
$$

Now, we have to verify that the conditions of the Banach fixed point Theorem are satisfied for the map $H$.

Step 1. There exist $R, T>0$ such that for any $f \in B_{R}^{T}$, it holds that $H(f) \in B_{R}^{T}$.

From (8), thanks to (5) and the embedding $W^{\beta, p}(\Omega) \hookrightarrow W^{1, p}(\Omega)$ we get

$$
\begin{aligned}
\|u(t)\|_{W^{1, p}} \leq C\left\|u_{0}\right\|_{W^{1, p}}+C \int_{0}^{t} & e^{-\nu(t-\tau)}(t-\tau)^{-\kappa}\left(\|F(f, v)\|_{W_{\mathcal{B}}^{2 \alpha-2, p}+}\right. \\
& \left.+\left\|A_{\alpha-1} \mathcal{B}^{c} \gamma g(f, v)\right\|_{W_{\mathcal{B}}^{2 \alpha-2, p}}\right) d \tau .
\end{aligned}
$$

Taking into account the embedding $W^{1-1 / p, p}(\partial \Omega) \hookrightarrow W^{2 \alpha-1-1 / p, p}(\partial \Omega)$ we have

$$
\begin{aligned}
\left\|A_{\alpha-1} \mathcal{B}^{c} \gamma g(f, v)\right\|_{W^{2 \alpha-2, p}(\partial \Omega)} & \leq C\|\gamma g(f, v)\|_{W^{2 \alpha-1-1 / p, p}(\partial \Omega)} \\
& \leq C\|\gamma g(f, v)\|_{W^{1-1 / p, p}(\partial \Omega)} \\
& \leq C\|g(f, v)\|_{W^{1, p}}
\end{aligned}
$$

also, having in mind $[2,(7.5),(7.8)]$, we have $L^{p}:=W_{\mathcal{B}}^{0, p} \hookrightarrow W_{\mathcal{B}}^{2 \alpha-2, p}$. Thus, we infer

$$
\|u(t)\|_{W^{1, p}} \leq C\left\|u_{0}\right\|_{W^{1, p}}+C \int_{0}^{t} e^{-\nu(t-\tau)}(t-\tau)^{-\kappa}\left(\|F(f, v)\|_{p}+\|g(f, v)\|_{W^{1, p}}\right) d \tau .
$$


On one hand we have

$$
\|F(f, v)\|_{L^{p}} \leq \chi\left(\|\nabla f \cdot \nabla v\|_{p}+\|f h(f)\|_{p}+\|f v\|_{p}\right)+\mu\left(\|f\|_{p}+\left\|f^{2}\right\|_{p}\right) .
$$

Now, we estimate each term of (10) separately

$$
\begin{aligned}
\|\nabla f \cdot \nabla v\|_{p} & \leq\|\nabla v\|_{\infty}\|\nabla f\|_{p} \\
& \leq C\|v\|_{W^{1, \infty}}\|f\|_{W^{1, p}} \\
& \leq C\|v\|_{W^{2, p}}\|f\|_{W^{1, p}} \\
& \leq C\left(\|h(f)\|_{p}+C\left(r^{\prime}\right)\right)\|f\|_{W^{1, p}} \\
& \leq C\left(\|h(f)\|_{\infty}+C\left(r^{\prime}\right)\right)\|f\|_{W^{1, p}} \\
& \leq C\left(\|f\|_{W^{1, p}}+C\left(r^{\prime}\right)\right)\|f\|_{W^{1, p}} .
\end{aligned}
$$

The remaining terms of (10) can be estimated in a similar way to obtain

$$
\|F(f, v)\|_{p} \leq C\left(\chi, \mu, r^{\prime},\|f\|_{W^{1, p}}\right)
$$

with $C\left(\chi, \mu, r^{\prime},\|f\|_{W^{1, p}}\right)$ an increasing function on its arguments. On the other hand we have

$$
\|g(f, v)\|_{W^{1, p}} \leq \frac{\chi \theta r^{\prime}}{2}\|f\|_{W^{1, p}}+\chi r^{\prime}\|f v\|_{W^{1, p}}+\|r \theta\|_{W^{1, p}}+r\|f\|_{W^{1, p}} .
$$

The term $\|f v\|_{W^{1, p}}$ is estimated as follows

$$
\begin{aligned}
\|f v\|_{W^{1, p}} & \leq C\|f\|_{W^{1, p}}\|v\|_{W^{1, \infty}} \\
& \leq C\|f\|_{W^{1, p}}\left(\|h(f)\|_{p}+C\left(r^{\prime}\right)\right) \\
& \leq C\|f\|_{W^{1, p}}\left(\|f\|_{W^{1, p}}+C\left(r^{\prime}\right)\right)
\end{aligned}
$$

So, we obtain

$$
\|g(f, v)\|_{W^{1, p}} \leq C\left(\chi, r, r^{\prime}, \theta,\|f\|_{W^{1, p}}\right),
$$

where $C\left(\chi, r, r^{\prime}, \theta,\|f\|_{W^{1, p}}\right)$ is an increasing function on its arguments. Now, we plug (11) and (13) in (9) to obtain

$$
\begin{aligned}
\|u(t)\|_{W^{1, p}} & \leq C\left\|u_{0}\right\|_{W^{1, p}}+C(R) \int_{0}^{t} C e^{-\nu(t-\tau)}(t-\tau)^{-\kappa} d \tau \\
& \leq C\left\|u_{0}\right\|_{W^{1, p}}+C(R) T^{1-\kappa} .
\end{aligned}
$$

Thus, choosing $R>C\left\|u_{0}\right\|_{W^{1, p}}$ and $\tau_{0}=T(R)$ sufficiently small then $\|u\|_{X_{\tau_{0}}} \leq R$. Moreover, $\|u\|_{X_{T}} \leq R$ for all $T \leq \tau_{0}$. Now, we fix $R>C\left\|u_{0}\right\|_{W^{1, p}}$ and $T \leq \tau_{0}$ is free to our disposal.

Step 2. H is contractive.

Let $f_{1}, f_{2} \in B_{R}^{T}$ then, $u_{1}=H\left(f_{1}\right) \in B_{R}^{T}$ and $u_{2}=H\left(f_{2}\right) \in B_{R}^{T}$ and

$u_{1}(t)-u_{2}(t)=\int_{0}^{t} e^{-(t-\tau) A_{\alpha-1}}\left(\left(F\left(f_{1}, v_{1}\right)-F\left(f_{2}, v_{2}\right)\right)+A_{\alpha-1} \mathcal{B}^{c} \gamma\left(g\left(f_{1}, v_{1}\right)-g\left(f_{2}, v_{2}\right)\right)\right) d \tau$.

So, we obtain

$$
\begin{aligned}
\left\|u_{1}(t)-u_{2}(t)\right\|_{W^{1, p}} \leq \int_{0}^{t} & e^{-\nu(t-\tau)}(t-\tau)^{-\kappa}\left(\left\|F\left(f_{1}, v_{1}\right)-F\left(f_{2}, v_{2}\right)\right\|_{p}+\right. \\
& \left.+\left\|g\left(f_{1}, v_{1}\right)-g\left(f_{2}, v_{2}\right)\right\|_{W^{1, p}}\right) d \tau
\end{aligned}
$$


On one hand we have

$$
\begin{aligned}
\left\|F\left(f_{1}, v_{1}\right)-F\left(f_{2}, v_{2}\right)\right\|_{p} \leq & \chi\left\|\nabla f_{2} \cdot \nabla v_{2}-\nabla f_{1} \cdot \nabla v_{1}\right\|_{p}+\chi\left\|f_{1} h\left(f_{1}\right)-f_{2} h\left(f_{2}\right)\right\|_{p}+ \\
& +\mu\left\|f_{1}-f_{2}\right\|_{p}+\mu\left\|f_{2}^{2}-f_{1}^{2}\right\|_{p} .
\end{aligned}
$$

Now, we estimate each term of (15) separately

$$
\begin{aligned}
\left\|\nabla f_{2} \cdot \nabla v_{2}-\nabla f_{1} \cdot \nabla v_{1}\right\|_{p} & \leq\left\|\nabla\left(f_{2}-f_{1}\right) \cdot \nabla v_{2}\right\|_{p}+\left\|\nabla f_{1} \cdot \nabla\left(v_{2}-v_{1}\right)\right\|_{p} \\
& \leq C\left(\left\|v_{2}\right\|_{W^{2, p}}\left\|f_{1}-f_{2}\right\|_{W^{1, p}}+R\left\|v_{1}-v_{2}\right\|_{W^{2, p}}\right) \\
& \leq C\left(C(R)\left\|f_{1}-f_{2}\right\|_{W^{1, p}}+R\left\|h\left(f_{1}\right)-h\left(f_{2}\right)\right\|_{p}\right) .
\end{aligned}
$$

Taking into account that $\left\|h\left(f_{1}\right)-h\left(f_{2}\right)\right\|_{p} \leq\left\|\left(f_{1}\right)_{+}-\left(f_{2}\right)_{+}\right\|_{\infty} \leq\left\|f_{1}-f_{2}\right\|_{\infty}$ we get

$$
\left\|\nabla f_{2} \cdot \nabla v_{2}-\nabla f_{1} \cdot \nabla v_{1}\right\|_{p} \leq C(R)\left\|f_{1}-f_{2}\right\|_{W^{1, p}} .
$$

For the remaining terms of (15) we can argue in a similar way to obtain

$$
\left\|F\left(f_{1}, v_{1}\right)-F\left(f_{2}, v_{2}\right)\right\|_{p} \leq C(R)\left\|f_{1}-f_{2}\right\|_{W^{1, p}} .
$$

On the other hand we have

$$
\left\|g\left(f_{1}, v_{1}\right)-g\left(f_{2}, v_{2}\right)\right\|_{W^{1, p}} \leq\left(\frac{\chi \theta r^{\prime}}{2}+r\right)\left\|f_{1}-f_{2}\right\|_{W^{1, p}}+\chi r^{\prime}\left\|f_{2} v_{2}-f_{1} v_{1}\right\|_{W^{1, p}} .
$$

We deduce

$$
\begin{aligned}
\left\|f_{2} v_{2}-f_{1} v_{1}\right\|_{W^{1, p}} & \leq\left\|v_{2}\left(f_{2}-f_{1}\right)\right\|_{W^{1, p}}+\left\|f_{1}\left(v_{2}-v_{1}\right)\right\|_{W^{1, p}} \\
& \leq\left\|v_{2}\right\|_{W^{1, \infty}}\left\|f_{1}-f_{2}\right\|_{W^{1, p}}+\left\|f_{1}\right\|_{W^{1, p}}\left\|v_{2}-v_{1}\right\|_{W^{1, \infty}} \\
& \leq C(R)\left\|f_{1}-f_{2}\right\|_{W^{1, p}} .
\end{aligned}
$$

Thus, we get

$$
\left\|g\left(f_{1}, v_{1}\right)-g\left(f_{2}, v_{2}\right)\right\|_{W^{1, p}} \leq C(R)\left\|f_{1}-f_{2}\right\|_{W^{1, p}} .
$$

Finally, we put the estimates (16) and (17) in (14) to obtain

$$
\left\|u_{1}-u_{2}\right\|_{X_{T}} \leq C(R) T^{1-\kappa}\left\|f_{1}-f_{2}\right\|_{X_{T}} .
$$

Hence, taking $T$ sufficiently small we prove that $H$ is contractive.

Now we deal with the regularity of the solution. Let us fix any $t \in(0, \tau)$ then the equation for $u$ has the abstract representation

$$
\frac{d u}{d t}+\left(I+A_{\alpha-1}\right) u=f(x, t), u(0)=u_{0} .
$$

Thus, thanks to $\left[9\right.$, Theorem 3.5.2] $\frac{d u}{d t}(t) \in X_{\kappa}$ with $\kappa<1$. In particular, by Lemma 2.2 , we have $\frac{d u}{d t}(t) \in W^{\beta, p}$ for some $\beta>1, p>d$. So, we obtain $u \in \mathcal{C}^{1}\left((0, \tau) ; W^{1, p}(\Omega)\right)$ and since the $v$-equation preserves the regularity in time then $v \in \mathcal{C}^{1}\left((0, \tau) ; W^{1, p}(\Omega)\right)$. We observe that

$$
\left\{\begin{aligned}
-\Delta v(t)+v(t) & =h(u)(t) & & \text { in } \Omega, \\
\frac{\partial v}{\partial n}(t)+r^{\prime} v(t) & =\frac{r^{\prime} \theta}{2} & & \text { on } \partial \Omega .
\end{aligned}\right.
$$


Taking into account that $h(u)(t) \in \mathcal{C}^{\alpha}(\bar{\Omega})$ then the elliptic regularity assures $v(t) \in$ $\mathcal{C}^{2+\alpha}(\bar{\Omega})$. So, we have proved that $v \in \mathcal{C}^{1}\left((0, \tau) ; \mathcal{C}^{2+\alpha}(\bar{\Omega})\right)$. Now we rewrite the $u$-equation as follows

$$
\begin{cases}-\Delta u(t)+\nabla u(t) \cdot \nabla v(t)=f(t) & \text { in } \Omega, \\ \frac{\partial u}{\partial n}(t)+\left(r-\chi r^{\prime}\left(\frac{\theta}{2}-v\right)\right) u(t)=r \theta & \text { on } \partial \Omega,\end{cases}
$$

where

$$
f(t):=\left(u h(u)-u v+\mu u(1-u)-u_{t}\right)(t) .
$$

Since $f(t) \in \mathcal{C}^{\alpha}(\bar{\Omega}), r-\chi r^{\prime}\left(\frac{\theta}{2}-v\right)(t) \in \mathcal{C}^{1+\alpha}(\partial \Omega)$ and $\nabla v(t) \in \mathcal{C}^{\alpha}(\bar{\Omega})$ then elliptic regularity entails $u(t) \in \mathcal{C}^{2+\alpha}(\bar{\Omega})$.

Next we observe that the positivity of $(u, v)$ is consequence of the maximum principle for parabolic equations.

At the end we show the continuity respect to the initial data, for this purpose we argue in the following manner. Let $R>C\left(\left\|u_{0}\right\|_{W^{1, p}}+\left\|\bar{u}_{0}\right\|_{W^{1, p}}\right)$. We have

$$
\begin{aligned}
u\left(u_{0}\right)(t) & =e^{-t A_{\alpha-1}} u_{0}+\int_{0}^{t} e^{-(t-\tau) A_{\alpha-1}}\left(F\left(u\left(u_{0}\right), v\left(u_{0}\right)\right)+A_{\alpha-1} \mathcal{B}^{c} \gamma g\left(u\left(u_{0}\right), v\left(u_{0}\right)\right)\right) d \tau \\
& =e^{-t A_{\alpha-1}} u_{0}+H\left(u\left(u_{0}\right)\right)-e^{-t A_{\alpha-1}} u_{0} .
\end{aligned}
$$

Hence, we infer

$$
\left\|\left(u\left(u_{0}\right)-u\left(\bar{u}_{0}\right)\right)(t)\right\|_{W^{1, p}} \leq 2\left\|e^{-t A_{\alpha-1}}\left(u_{0}-\bar{u}_{0}\right)\right\|_{W^{1, p}}+\| H\left(u\left(u_{0}\right)-H\left(u\left(\bar{u}_{0}\right)\right) \|_{W^{1, p}} .\right.
$$

Taking supremum on time, thanks to the contractivity of $\mathrm{H}$, we obtain

$$
\left\|u\left(u_{0}\right)-u\left(\bar{u}_{0}\right)\right\|_{X_{T}} \leq C\left\|u_{0}-\bar{u}_{0}\right\|_{W^{1, p}}+k\left\|u\left(u_{0}\right)-u\left(\bar{u}_{0}\right)\right\|_{X_{T}},
$$

with $k<1$. Also we have

$$
\begin{aligned}
\left\|\left(v\left(u_{0}\right)-v\left(\bar{u}_{0}\right)\right)(t)\right\|_{W^{2, p}} & \leq C(t)\left\|h\left(u\left(u_{0}\right)\right)(t)-h\left(\bar{u}_{0}\left(u_{0}\right)\right)(t)\right\|_{L^{p}} \\
& \leq C(t)\left\|u\left(u_{0}\right)-u\left(\bar{u}_{0}\right)\right\|_{W^{1, p}} .
\end{aligned}
$$

From (18) the proof of the continuity can be easily concluded.

Now we deal with the issue of global in time solutions. To this end we have just to show that $\|u(t)\|_{W^{1, p}} \leq C(t)$ for all $t<T_{\max }$ where $T_{\max }$ stands for the maximal interval of existence. We observe that

$$
\begin{cases}-\Delta v+v=\frac{u}{1+u} & \text { in } \Omega \times\left(0, T_{\max }\right), \\ \frac{\partial v}{\partial n}+r^{\prime} v=\frac{r^{\prime} \theta}{2} & \text { on } \partial \Omega \times\left(0, T_{\max }\right) .\end{cases}
$$

Since $\left\|\frac{u(t)}{1+u(t)}\right\|_{\infty} \leq 1$ then $\|v(t)\|_{W^{2, p}} \leq C$ for all $t \in\left[0, T_{\max }\right)$. Next we put the formula of generalized variations of constants to obtain

$$
u(t)=e^{-t A_{\alpha-1}} u_{0}+\int_{0}^{t} e^{-(t-\tau) A_{\alpha-1}}\left(F(u, v)+A_{\alpha-1} \mathcal{B}^{c} g(u, v)\right) d \tau .
$$


So,

$$
\begin{aligned}
\|u(t)\|_{W^{1, p}} & \leq C\left\|u_{0}\right\|_{W^{1, p}}+C \int_{0}^{t} e^{-\nu(t-\tau)}(t-\tau)^{-\kappa}\left(\|F(u, v)\|_{L^{p}}+\|g(u, v)\|_{W^{1, p}}\right) \\
& \leq C\left\|u_{0}\right\|_{W^{1, p}}+C \int_{0}^{t} e^{-\nu(t-\tau)}(t-\tau)^{-\kappa}\|u(\tau)\|_{W^{1, p}} d \tau
\end{aligned}
$$

Finally the proof of global existence concludes with use of Gronwall's Lemma.

\section{Asymptotic behavior for $\theta=1$}

When $\theta=1$ there exists the coexistence state $(u, v)=(1,1 / 2)$.

\subsection{Case $r^{\prime}>0$}

Theorem 4.1 Assume $r>0$. There exists $C\left(r, r^{\prime}\right)>0$ a function increasing on $r$ such that if $0<\chi<C\left(r, r^{\prime}\right)$ then

$$
\|u(t)-1\|_{W^{\beta, p}} \leq C e^{-\alpha(\beta, p, \chi) t} \quad\|v(t)-1 / 2\|_{W^{2, p}} \leq C e^{-\gamma(p, \chi) t}
$$

where $\beta<1, p \geq 2$ and $\alpha(\beta, p, \chi)>0, \gamma(p, \chi)>0$ are given functions that can be computed.

Proof. We multiply the $u$-equation by $u-1$ and after integrating in the spatial variable we get

$$
\begin{aligned}
\frac{d}{2 d t} \int_{\Omega}(u-1)^{2}= & -\int_{\Omega}|\nabla(u-1)|^{2}+\chi \int_{\Omega} u \nabla v \cdot \nabla(u-1)- \\
& -r \int_{\partial \Omega}(u-1)^{2}-\mu \int_{\Omega} u(u-1)^{2} \\
= & -\int_{\Omega}|\nabla(u-1)|^{2}+\frac{\chi}{2} \int_{\Omega} \nabla v \cdot \nabla(u-1)^{2}+\chi \int_{\Omega} \nabla v \cdot \nabla(u-1)- \\
& -r \int_{\partial \Omega}(u-1)^{2}-\mu \int_{\Omega} u(u-1)^{2} .
\end{aligned}
$$

Now, we compute the terms $\int_{\Omega} \nabla v \cdot \nabla(u-1)^{2}, \int_{\Omega} \nabla v \cdot \nabla(u-1)$. For this purpose we multiply the $v$-equation by $u-1$ and we integrate in $\Omega$ to obtain

$$
\begin{aligned}
\int_{\Omega} \nabla v & \cdot \nabla(u-1)=\int_{\Omega}\left(\frac{u}{1+u}-v\right)(u-1)+r^{\prime} \int_{\partial \Omega}\left(\frac{1}{2}-v\right)(u-1) \\
& =\int_{\Omega}\left(\frac{u}{1+u}-\frac{1}{2}\right)^{2}(u-1)+\int_{\Omega}\left(\frac{1}{2}-v\right)(u-1)+r^{\prime} \int_{\partial \Omega}\left(\frac{1}{2}-v\right)(u-1) \\
& =\int_{\Omega} \frac{(u-1)^{2}}{2(1+u)}+\int_{\Omega}\left(\frac{1}{2}-v\right)(u-1)+r^{\prime} \int_{\partial \Omega}\left(\frac{1}{2}-v\right)(u-1) .
\end{aligned}
$$

In the same manner we have

$$
\int_{\Omega} \nabla v \cdot \nabla(u-1)^{2}=\int_{\Omega} \frac{(u-1)^{3}}{2(1+u)}+\int_{\Omega}\left(\frac{1}{2}-v\right)(u-1)^{2}+r^{\prime} \int_{\partial \Omega}\left(\frac{1}{2}-v\right)(u-1)^{2} .
$$

Now, we estimate each term in the right-hand side of (21) and (20). We know that $\frac{s-1}{2(1+s)}<\frac{1}{2}$ for all $s \geq 0$. Therefore,

$$
\int_{\Omega} \frac{(u-1)^{3}}{2(1+u)} \leq \frac{1}{2} \int_{\Omega}(u-1)^{2}
$$


Since $v \geq 0$ then

$$
\int_{\Omega}\left(\frac{1}{2}-v\right)(u-1)^{2} \leq \frac{1}{2} \int_{\Omega}(u-1)^{2}, \quad r^{\prime} \int_{\partial \Omega}\left(\frac{1}{2}-v\right)(u-1)^{2} \leq \frac{r^{\prime}}{2} \int_{\partial \Omega}(u-1)^{2} .
$$

By the positivity of $u$ we have

$$
\int_{\Omega} \frac{(u-1)^{2}}{2(1+u)} \leq \frac{1}{2} \int_{\Omega}(u-1)^{2}
$$

It remains to estimate $(I)=\int_{\Omega}\left(\frac{1}{2}-v\right)(u-1)+r^{\prime} \int_{\partial \Omega}\left(\frac{1}{2}-v\right)(u-1)$. We know that

$$
(I) \leq(I I)+\frac{1}{2} \int_{\Omega}(u-1)^{2}+\frac{r^{\prime}}{2} \int_{\partial \Omega}(u-1)^{2},
$$

where

$$
(I I)=\frac{1}{2} \int_{\Omega}\left(\frac{1}{2}-v\right)^{2}+\frac{r^{\prime}}{2} \int_{\partial \Omega}\left(\frac{1}{2}-v\right)^{2} .
$$

Now, we try to estimate $(I I)$, to this aim we subtract $1 / 2$ in the $v$-equation and we multiply it by $v-1 / 2$ to get, after integrating in the spatial variable, that

$\int_{\Omega}\left|\nabla\left(v-\frac{1}{2}\right)\right|^{2}+\frac{1}{2} \int_{\Omega}\left(v-\frac{1}{2}\right)^{2}+\frac{r^{\prime}}{2} \int_{\partial \Omega}\left(\frac{1}{2}-v\right)^{2}+(I I)=\int_{\Omega}\left(\frac{u}{1+u}-\frac{1}{2}\right)\left(v-\frac{1}{2}\right)$.

We provide a bound of the first and third term in the above inequality with the following argument. Let $\lambda_{1}\left(r^{\prime} / 2\right) \in\left[0, \lambda_{1}(+\infty)\right)$ the principal eigenvalue of

$$
\begin{cases}-\Delta \varphi=\lambda \varphi & \text { in } \Omega, \\ \frac{\partial \varphi}{\partial n}+\frac{r^{\prime}}{2} \varphi=0 & \text { on } \partial \Omega .\end{cases}
$$

By the variational characterization of $\lambda_{1}\left(r^{\prime} / 2\right)$ we have that for all $\varphi \in H^{1}(\Omega)$

$$
\lambda_{1}\left(r^{\prime} / 2\right) \int_{\Omega} \varphi^{2} \leq \int_{\Omega}|\nabla \varphi|^{2}+\frac{r^{\prime}}{2} \int_{\partial \Omega} \varphi^{2} .
$$

Putting the previous estimate in (22) we obtain

$$
\left(\frac{1}{2}+\lambda_{1}\left(r^{\prime} / 2\right)\right) \int_{\Omega}\left(v-\frac{1}{2}\right)^{2}+(I I) \leq \epsilon \int_{\Omega} \frac{(u-1)^{2}}{4(1+u)^{2}}+\frac{1}{4 \epsilon} \int_{\Omega}\left(v-\frac{1}{2}\right)^{2},
$$

for all $\epsilon>0$. We pick $\epsilon=\epsilon_{0}>0$ such that $\frac{1}{4 \epsilon_{0}} \leq \frac{1}{2}+\lambda_{1}\left(r^{\prime} / 2\right)$. Thus,

$$
(I I) \leq \frac{\epsilon_{0}}{4} \int_{\Omega}(u-1)^{2}
$$

All the previous estimates allow us to infer from (19) that

$$
\frac{1}{2} \frac{d}{d t} \int_{\Omega}(u-1)^{2} \leq-\int_{\Omega}|\nabla(u-1)|^{2}+\frac{\chi\left(6+\epsilon_{0}\right)}{4} \int_{\Omega}(u-1)^{2}+\left(\frac{3 \chi r^{\prime}}{4}-r\right) \int_{\partial \Omega}(u-1)^{2} .
$$


Let $z(\chi)=r-\frac{3 \chi r^{\prime}}{4}$. Observe that, by the variational characterization of $\lambda_{1}(z(\chi))$ we have

$$
\frac{1}{2} \frac{d}{d t} \int_{\Omega}(u-1)^{2} \leq F(\chi) \int_{\Omega}(u-1)^{2},
$$

where

$$
F(\chi):=\frac{\chi\left(6+\epsilon_{0}\right)}{4}-\lambda_{1}(z(\chi)) .
$$

By the monotonicity of $z$ and $\lambda_{1}$ we have that $F(\chi)$ is increasing in $\chi$. Since $F(0)<0$ and $F\left((4 r) /\left(3 r^{\prime}\right)\right)>0$ then there exists a unique $\chi^{*}=C\left(r, r^{\prime}\right)$ such that for all $\chi<C\left(r, r^{\prime}\right)$ $F(\chi)<0$. From here we deduce that

$$
\|u(t)-1\|_{2}^{2} \leq\left\|u_{0}-1\right\|_{2}^{2} e^{2 F(\chi) t} .
$$

Let $p \geq 2$. Since $\|u(t)\|_{W^{1, p}} \leq C$ for all $t \geq 0$ then

$$
\int_{\Omega}(u-1)^{p} \leq \int_{\Omega}(u-1)^{p-2}(u-1)^{2} \leq C^{p-2}\left\|u_{0}-1\right\|_{2}^{2} e^{2 F(\chi) t} .
$$

Therefore,

$$
\|u(t)-1\|_{p} \leq C^{p-2}\left\|u_{0}-1\right\|_{2}^{2 / p} e^{2 F(\chi) / p t} .
$$

Next, the Gagliardo-Nirenberg inequality (see for instance [9, p. 37]) entails

$$
\|u(t)-1\|_{W^{\beta, p}} \leq C\|u(t)-1\|_{W^{1, p}}^{\kappa}\|u(t)-1\|_{p}^{1-\kappa}
$$

for $\beta<\kappa, \kappa \in(0,1)$. From the last inequality easily follows the convergence of $u$ to 1 in norm $W^{\beta, p}$. Finally the convergence for $v$ follows from the $W^{2, p}$ estimates of $v$.

Remark 4.2 Observe that if $\mu=r=0$ integrating in the $u$-equation we get

$$
\int_{\Omega} u(t)=\int_{\Omega} u_{0}
$$

and then we can assure in general that $u$ tends to 1.

\subsection{Case $r^{\prime}=0$}

In this case we follow the lines of [11].

Theorem 4.3 We assume $\min _{x \in \bar{\Omega}} u_{0}(x)>0$ and consider the constant $\gamma_{0}$ defined as follows

$$
\gamma_{0}:=\frac{2 \chi}{\left(1+\frac{\underline{\underline{u}}_{0}}{\bar{u}_{0}}\right)^{2}}-\mu<0
$$

where

$$
\bar{u}_{0}:=\max \left\{\max _{x \in \bar{\Omega}} u_{0}(x), 1\right\}, \quad \underline{u}_{0}:=\min \left\{\min _{x \in \bar{\Omega}} u_{0}(x), 1\right\},
$$

then the solution $(u, v)$ to (2) fulfills

$$
\|u(t)-1\|_{\infty}+\left\|v(t)-\frac{1}{2}\right\|_{W^{2, p}} \leq-C \epsilon_{0}^{-1} \ln \left(\epsilon_{0}\right) e^{\gamma_{0} \epsilon_{0} t}, \quad t>0
$$

for any $p>1$ and $\epsilon_{0}:=\frac{\underline{u}_{0}}{\bar{u}_{0}}$. 
Proof. Let

$$
\begin{aligned}
& F_{1}(u, v):=\chi u\left(\frac{u}{1+u}-\frac{v}{1+v}\right)+\mu u(1-u) \\
& F_{2}(u, v):=\chi v\left(\frac{v}{1+v}-\frac{u}{1+u}\right)+\mu v(1-v)
\end{aligned}
$$

and $(\bar{u}, \underline{u})=(\bar{u}(t), \underline{u}(t))$ the solution to the following system of differential equations

$$
\left\{\begin{array}{l}
\bar{u}_{t}=F_{1}(\bar{u}, \underline{u}), \\
\underline{u}_{t}=F_{2}(\bar{u}, \underline{u}),
\end{array}\right.
$$

with initial data $(\bar{u}(0), \underline{u}(0))=\left(\bar{u}_{0}, \underline{u}_{0}\right)$. Let us decompose the proof of the Theorem into several steps.

Step 1. We first claim that

$$
0<\underline{u} \leq \bar{u} \quad \text { in }\left(0, T_{\max }\right) .
$$

where $T_{\max }>0$ denotes the maximal existence time of (25). Having in mind that $F_{1}$ and $F_{2}$ are regular functions then, at least locally the system (25) has a unique solution in $\left(0, T_{\max }\right)$. Since $F_{1}(0, \underline{u})=0$ and $\bar{u}_{0}>0$ then $\bar{u}(t)>0$ for all $t \in\left(0, T_{\max }\right)$. Arguing in the same manner we can prove that $\underline{u}(t)>0$ for all $t \in\left(0, T_{\text {max }}\right)$. Finally, it remains to prove $\underline{u} \leq \bar{u}$. Suppose that there exists $t_{0} \geq 0$ such that $\bar{u}\left(t_{0}\right)=\underline{u}\left(t_{0}\right)$; then by the uniqueness $\bar{u}(t)=\underline{u}(t)=v(t)$ for all $t \geq 0$ where $v$ solves $v_{t}=\mu v(1-v), v\left(t_{0}\right)=\bar{u}\left(t_{0}\right)$. So, if $\underline{u}_{0}=\bar{u}_{0}$, then $\underline{u}=\bar{u}$ in $\left(0, T_{\max }\right)$; if $\underline{u}_{0}<\bar{u}_{0}$, the $\underline{u}<\bar{u}$ in $\left(0, T_{\max }\right)$, because the previous $t_{0}$ can not exist.

Step 2. We have

$$
\underline{u} \leq 1 \leq \bar{u} \quad \text { in }\left(0, T_{\max }\right) .
$$

Since $\frac{x}{x+1}$ is an increasing function, by (25) and Step $1, \bar{u}$ satisfies $\bar{u}_{t} \geq \mu \bar{u}(1-\bar{u})$. Since $\bar{u}(0) \geq 1$, by comparison we get $\bar{u} \geq 1$. In the same fashion we prove $\underline{u} \leq 1$.

Step 3. We next show that under assumption (23) we have

$$
\bar{u}(t)-\underline{u}(t) \leq-\epsilon_{0}^{-1} \ln \left(\epsilon_{0}\right) e^{\gamma_{0} \epsilon_{0} t} .
$$

On multiplying the first equation in (25) by $\frac{1}{\bar{u}}$ and the second one by $\frac{1}{\underline{u}}$, we obtain

$$
\frac{\bar{u}_{t}}{\bar{u}}=\chi\left(\frac{\bar{u}}{1+\bar{u}}-\frac{\underline{u}}{1+\underline{u}}\right)+\mu(1-\bar{u}) \quad \text { in }\left(0, T_{\max }\right),
$$

and

$$
\frac{\underline{u}_{t}}{\underline{u}}=\chi\left(\frac{\underline{u}}{1+\underline{u}}-\frac{\bar{u}}{1+\bar{u}}\right)+\mu(1-\underline{u}) \quad \text { in }\left(0, T_{\max }\right),
$$

for $t>0$, respectively. Subtracting the previous equations we get

$$
\frac{d}{d t}\left(\ln \frac{\bar{u}}{\underline{u}}\right)=2 \chi\left(\frac{\bar{u}}{1+\bar{u}}-\frac{\underline{u}}{1+\underline{u}}\right)-\mu(\bar{u}-\underline{u}) .
$$


Next, we observe that the mean value theorem entails

$$
2 \chi\left(\frac{\bar{u}}{1+\bar{u}}-\frac{\underline{u}}{1+\underline{u}}\right)=\frac{2 \chi}{(1+\xi(t))^{2}}(\bar{u}-\underline{u}),
$$

where $\xi(t) \in(\underline{u}(t), \bar{u}(t))$. Let

$$
\gamma(t):=\left(\frac{2 \chi}{(1+\xi(t))^{2}}-\mu\right)
$$

Therefore

$$
\frac{d}{d t}\left(\ln \frac{\bar{u}}{\underline{u}}\right)=\gamma(t)(\bar{u}-\underline{u}) .
$$

Next, we claim that $\underline{u}(t) \geq \epsilon_{0}$. Suppose the contrary then the set

$$
A:=\left\{t \in\left[0, T_{\max }\right): \underline{u}(t)<\epsilon_{0}\right\}
$$

in not empty and bounded from below because $0 \notin A$. Therefore, there exists $t_{0}:=\inf A$. It is not difficult to infer that $t_{0}>0$. Thanks to $(23)$ there exists $k>0$ such that

$$
\frac{2 \chi}{\left(1+\epsilon_{0}-k\right)^{2}}-\mu \leq 0
$$

By the continuity of $\underline{u}$ and the definition of $t_{0}$ we have that there exists $\delta_{0}(k)>0$ such that $\underline{u}\left(t_{0}+\delta\right)>\epsilon-k$ for all $\delta \leq \delta_{0}$. More generally, we have $\underline{u}(t)>\epsilon_{0}-k$ for all $t \in\left[0, t_{0}+\delta_{0}\right]$. Since $\xi(t) \geq \underline{u}(t) \geq \epsilon_{0}-k$ for all $t \in\left[0, t_{0}+\delta_{0}\right]$ then $\gamma(t) \leq 0$ for all $t \in\left[0, t_{0}+\delta_{0}\right]$. Hence, from (31) we get

$$
\frac{\bar{u}(t)}{\underline{u}(t)} \leq \frac{\bar{u}(0)}{\underline{u}(0)}=\epsilon_{0}^{-1}, \quad \forall t \in\left[0, t_{0}+\delta_{0}\right] .
$$

Therefore, taking into account that $\bar{u}(t) \geq 1$, the previous inequality asserts

$$
\epsilon_{0} \leq \underline{u}(t) \forall t \in\left[0, t_{0}+\delta_{0}\right] .
$$

From (32) we infer inf $A \geq t_{0}+\delta_{0}$, a contradiction. As a consequence of the previous proof we have that $\gamma(t) \leq \gamma_{0}<0$. Now, we observe that the mean value theorem entails

$$
\bar{u}-\underline{u}=e^{\ln \bar{u}}-e^{\ln \underline{u}}=e^{\ln \hat{u}(t)}(\ln \bar{u}-\ln \underline{u})=\hat{u}(t) \ln \left(\frac{\bar{u}}{\underline{u}}\right),
$$

where $\hat{u}(t) \in(\underline{u}(t), \bar{u}(t))$. Substituting the term (33) in (31) we deduce

$$
\frac{d}{d t}\left(\ln \left(\frac{\bar{u}}{\underline{u}}\right)\right)=\gamma(t) \hat{u}(t) \ln \left(\frac{\bar{u}}{\underline{u}}\right) \leq \gamma_{0} \epsilon_{0} \ln \left(\frac{\bar{u}}{\underline{u}}\right) .
$$

Upon integration this yields

$$
\ln \left(\frac{\bar{u}(t)}{\underline{u}(t)}\right) \leq-\ln \left(\epsilon_{0}\right) e^{\gamma_{0} \epsilon_{0} t} .
$$

The above inequality prove that $T_{\max }=+\infty$. Moreover substituting the estimate (33) in the previous inequality we get

$$
\bar{u}(t)-\underline{u}(t) \leq-\epsilon_{0}^{-1} \ln \left(\epsilon_{0}\right) e^{\gamma_{0} \epsilon_{0} t} .
$$


Step 4. We now establish a connection between (25) and (23) by showing that

$$
\underline{u}(t) \leq u(x, t) \leq \bar{u}(t) \quad \forall(x, t) \in \bar{\Omega} \times(0, \infty) .
$$

We consider $\bar{U}(x, t)=u(x, t)-\bar{u}(t)$ and $\underline{U}(x, t)=u(x, t)-\underline{u}(t)$ which satisfy:

$\bar{U}_{t}-\Delta \bar{U}=-\chi \nabla \cdot(\bar{U} \nabla v)-\chi \bar{u}\left(\frac{\bar{u}}{1+\bar{u}}-\frac{\underline{u}}{\underline{u}+1}\right)+\chi \bar{u}\left(\frac{u}{1+u}-v\right)+\mu u(1-u)-\mu \bar{u}(1-\bar{u})$ since

$$
\begin{aligned}
-\chi \bar{u}\left(\frac{\bar{u}}{1+\bar{u}}-\frac{\underline{u}}{\underline{u}+1}\right) & +\chi \bar{u}\left(\frac{u}{1+u}-v\right)=\chi \bar{u}\left(\frac{u}{1+u}-\frac{\bar{u}}{1+\bar{u}}+\frac{\underline{u}}{\underline{u}+1}-v\right), \\
& =\chi \bar{u}\left(\frac{1}{\left(1+\xi_{1}\right)^{2}} \bar{U}+\frac{\underline{u}}{\underline{u}+1}-v\right),
\end{aligned}
$$

and

$$
\mu u(1-u)-\mu \bar{u}(1-\bar{u})=\mu(1-\bar{u}-u) \bar{U}
$$

where $\xi_{1}(x, t) \in(\min \{u, \bar{u}\}, \max \{u, \bar{u}\})$. Then $\bar{U}$ satisfies

$$
\bar{U}_{t}-\Delta \bar{U}=-\chi \nabla \cdot(\bar{U} \nabla v)+\chi \bar{u}\left(\frac{1}{\left(1+\xi_{1}\right)^{2}} \bar{U}+\frac{\underline{u}}{\underline{u}+1}-v\right)+\mu(1-\bar{u}-u) \bar{U}
$$

i.e.

$$
\bar{U}_{t}-\Delta \bar{U}=-\chi \nabla \cdot(\bar{U} \nabla v)+\bar{U}\left(\frac{\chi \bar{u}}{\left(1+\xi_{1}\right)^{2}}+\mu(1-\bar{u}-u)\right)+\chi \bar{u}\left(\frac{\underline{u}}{\underline{u}+1}-v\right) .
$$

Notice that

$$
\begin{aligned}
& \int_{\Omega}(-\Delta \bar{U}+\chi \nabla \cdot(\bar{U} \nabla v)) \bar{U}_{+}= \\
= & \int_{\Omega}\left|\nabla \bar{U}_{+}\right|^{2}-\chi \int_{\Omega} \bar{U} \nabla v \cdot \nabla \bar{U}_{+}-\int_{\partial \Omega}\left(\frac{\partial \bar{U}}{\partial n}-\chi \bar{U} \frac{\partial v}{\partial n}\right) \bar{U}_{+} \\
= & \int_{\Omega}\left|\nabla \bar{U}_{+}\right|^{2}-\frac{\chi}{2} \int_{\Omega} \nabla v \cdot \nabla \bar{U}_{+}^{2}-\int_{\partial \Omega}\left(r(1-u)+\chi \bar{u} \frac{\partial v}{\partial n}\right)_{U_{+}} \\
= & \int_{\Omega}\left|\nabla \bar{U}_{+}\right|^{2}-\frac{\chi}{2} \int_{\Omega}\left(\frac{u}{1+u}-v\right) \bar{U}_{+}^{2}-\frac{\chi}{2} \int_{\partial \Omega} \frac{\partial v}{\partial n} \bar{U}_{+}^{2}-\int_{\partial \Omega}\left(r(1-u)+\chi \bar{u} \frac{\partial v}{\partial n}\right) \bar{U}_{+} \\
= & \int_{\Omega}\left|\nabla \bar{U}_{+}\right|^{2}+\frac{\chi}{2} \int_{\Omega}\left(v-\frac{u}{1+u}\right) \bar{U}_{+}^{2}-\int_{\partial \Omega} r(1-u) U_{+} .
\end{aligned}
$$

Since $\bar{U}_{+} \neq 0$ whenever $u \geq \bar{u}$ then by step $2, u \geq \bar{u} \geq 1$. As a consequence, the boundary term is non-positive. We take $\bar{U}_{+}$as test function in (35) to obtain

$$
\frac{d}{2 d t} \int_{\Omega} \bar{U}_{+}^{2}+\int_{\Omega}\left|\nabla \bar{U}_{+}\right|^{2} \leq \int_{\Omega} \bar{U}_{+}^{2} g(u, v, \bar{u})+\chi \int_{\Omega} \bar{U}_{+} \bar{u}\left(\frac{\bar{u}}{1+\bar{u}}-v\right),
$$

with

$$
g(u, v, \bar{u}):=\frac{\chi}{2}\left(\frac{u}{1+u}-v\right)+\frac{\chi \bar{u}}{\left(1+\xi_{1}\right)^{2}}+\mu(1-\bar{u}-u) .
$$

We try to estimate the last term in the right hand side of (36). We observe

$$
\begin{aligned}
\chi \int_{\Omega} \bar{U}_{+} \bar{u}\left(\frac{\underline{u}}{1+\underline{u}}-v\right) & \leq \chi \int_{\Omega} \bar{U}_{+} \bar{u}\left(\frac{\underline{u}}{1+\underline{u}}-v\right)_{+} \\
& \leq \chi \epsilon_{0}^{-1}\left(\int_{\Omega} \bar{U}_{+}^{2}+\int_{\Omega}\left(v-\frac{\underline{u}}{1+\underline{u}}\right)_{-}^{2}\right),
\end{aligned}
$$


where in the last inequality we used that $f_{+}=-(-f)_{-}$. We note that

$$
-\Delta v+v-\frac{\underline{u}}{1+\underline{u}}=\underline{U} \frac{1}{\left(1+\xi_{2}\right)^{2}}
$$

for some $\xi_{2}(x, t) \in(\min \{\underline{u}, u\}, \max \{\underline{u}, u\})$. After multiplying the previous expression by $\left(v-\frac{u}{1+\underline{u}}\right)$ - we get

$$
\begin{aligned}
\int_{\Omega}\left|\nabla\left(v-\frac{\underline{u}}{1+\underline{u}_{-}}\right)\right|^{2}+\int_{\Omega}\left(v-\frac{\underline{u}}{1+\underline{u}}\right)_{-}^{2} & =\int_{\Omega} \underline{U} \frac{1}{\left(1+\xi_{2}\right)^{2}}\left(v-\frac{\underline{u}}{1+\underline{u}}\right)_{-} \\
& \leq \int_{\Omega} \underline{U}_{-} \frac{1}{\left(1+\xi_{2}\right)^{2}}\left(v-\frac{\underline{u}}{1+\underline{u}}\right)_{\overline{2}} \\
& \leq \frac{1}{2} \int_{\Omega} \underline{U}_{-}^{2}+\frac{1}{2} \int_{\Omega}\left(v-\frac{\underline{u}}{1+\underline{u}}\right)_{-}
\end{aligned}
$$

Taking into account that the boundary term is non-positive then, from the above inequality we deduce

$$
\frac{1}{2} \int_{\Omega}\left(v-\frac{\underline{u}}{1+\underline{u}}\right)_{-}^{2} \leq \frac{1}{2} \int_{\Omega} \underline{U}_{-}^{2} .
$$

Plugging the estimate (38) into (37) we obtain

$$
\chi \int_{\Omega} \bar{U}_{+}\left(\frac{\underline{u}}{1+\underline{u}}-v\right) \leq \chi \epsilon_{0}^{-1}\left(\int_{\Omega} \bar{U}_{+}^{2}+\int_{\Omega} \underline{U}_{-}^{2}\right) .
$$

The previous inequality provides with the following bound in (36)

$$
\frac{d}{2 d t} \int_{\Omega} \bar{U}_{+}^{2} \leq \int_{\Omega} \bar{U}_{+}^{2} g(u, v, \bar{u})+\chi \epsilon_{0}^{-1}\left(\int_{\Omega} \bar{U}_{+}^{2}+\int_{\Omega} \underline{U}_{-}^{2}\right) .
$$

Since $g(u, v, \bar{u}) \leq C$ then

$$
\frac{d}{2 d t} \int_{\Omega} \bar{U}_{+}^{2} \leq C\left(\int_{\Omega} \bar{U}_{+}^{2}+\int_{\Omega} \underline{U}_{-}^{2}\right) .
$$

In the same fashion we have

$$
\frac{d}{2 d t} \int_{\Omega} \underline{U}_{-}^{2} \leq C\left(\int_{\Omega} \bar{U}_{+}^{2}+\int_{\Omega} \underline{U}_{-}^{2}\right) .
$$

Adding the above inequalities and taking into account that $\left(\bar{U}_{0}\right)_{+}=\left(\underline{U}_{0}\right)_{-}=0$, we may invoke Gronwall's Lemma to achieve

$$
\bar{U}_{+}=\underline{U}_{-}=0
$$

which proves the step.

Step 5. Since $\underline{u} \leq u \leq \bar{u}$ and $\underline{u} \leq 1 \leq \bar{u}$ then

$$
\|u(t)-1\|_{\infty} \leq \bar{u}-\underline{u} \leq-\epsilon_{0}^{-1} \ln \left(\epsilon_{0}\right) e^{\gamma_{0} \epsilon_{0} t} \forall t>0 .
$$


Next we observe that $\xi=\frac{1}{2}$ is the unique solution to the problem

$$
\begin{cases}-\Delta \xi+\xi=\frac{1}{2} & \text { in } \Omega \times(0,+\infty), \\ \frac{\partial \xi}{\partial n}=0 & \text { on } \partial \Omega \times(0,+\infty) .\end{cases}
$$

Therefore $z:=v-\xi$ satisfies

$$
\begin{cases}-\Delta z+z=\frac{u}{1+u}-\frac{1}{2} & \text { in } \Omega \times(0,+\infty), \\ \frac{\partial z}{\partial n}=0 & \text { on } \partial \Omega \times(0,+\infty),\end{cases}
$$

and elliptic regularity asserts

$$
\|z(t)\|_{W^{2, p}} \leq C\left\|\frac{u(t)-1}{2(1+u(t))}\right\|_{\infty} \leq C\|u(t)-1\|_{\infty},
$$

concluding the result.

Corollary 4.4 If $\mu \geq 2 \chi$ then the solution $(u, v)$ to (2) is globally exponentially asymptotically stable and converges to the homogeneous steady-state $\left(1, \frac{1}{2}\right)$. Moreover, if $\mu \geq 2 \chi$, then the previous homogeneous steady-state is the only positive solution to the steady-state problem associated to (2).

Proof. Assume min $u_{0}=0$ then, by the strong maximum principle, $\min u(\tau)>0$ for arbitrary $\tau>0$ small as desired. Next, we observe that $\gamma_{0}<2 \chi-\mu \leq 0$ and thanks to Theorem 4.2 we conclude the first part. The second part is a direct consequence of the global stability.

Another consequence of Theorem 4.2 is the next Corollary

Corollary 4.5 If $\mu>\frac{\chi}{2}$ then the solution $\left(1, \frac{1}{2}\right)$ to (2) is locally exponentially asymptotically stable.

\section{The stationary problem}

In this section, we analyze the stationary problem associated to (2), that is

$$
\begin{cases}-\Delta u=-\chi \nabla \cdot(u \nabla v)+\mu u(1-u) & \text { in } \Omega, \\ -\Delta v=-v+\frac{u}{1+u} & \text { in } \Omega, \\ \frac{\partial u}{\partial n}-\chi u \frac{\partial v}{\partial n}=r(\theta-u) & \text { on } \partial \Omega \\ \frac{\partial v}{\partial n}=r^{\prime}\left(\frac{\theta}{2}-v\right) & \text { on } \partial \Omega .\end{cases}
$$

First, in order to present and prove our main result, we need introduce some notation. Given functions $a, b \in \mathcal{C}(\bar{\Omega})$ with $a \geq a_{0}>0, r \in \mathcal{C}(\partial \Omega)$, we denote by $\lambda_{1}(a ; b ; N+r)$ the principal eigenvalue of the problem

$$
\begin{cases}-\operatorname{div}(a(x) \nabla u)=\lambda b(x) u & \text { in } \Omega, \\ \frac{\partial u}{\partial n}+r(x) u=0 & \text { on } \partial \Omega .\end{cases}
$$


Recall that $\lambda_{1}(a ; b ; N+r)$ is increasing in $a$ and $r$ and decreasing in $b$.

On the other hand, denote by $v_{r^{\prime}}$ the unique positive solution of

$$
\begin{cases}-\Delta v+v=1 & \text { in } \Omega, \\ \frac{\partial v}{\partial n}+r^{\prime} v=r^{\prime} \frac{\theta}{2} & \text { on } \partial \Omega .\end{cases}
$$

It is clear that $v_{r^{\prime}}=1$ if $r^{\prime}=0$. Moreover, if $r^{\prime}>0$

$$
\min \{1, \theta / 2\} \leq v_{r^{\prime}} \leq \max \{1, \theta / 2\}
$$

Finally, observe that since $u /(u+1) \leq 1$ we get

$$
v \leq v_{r^{\prime}} \quad \text { in } \Omega
$$

Now, we are ready to state our main result:

Theorem 5.1 a) Assume that $\theta>0$ and $r^{\prime} \geq 0$. Then, there exists at least a positive solution of (42) if $\mu \geq 0$ and $r \geq 0$.

b) Assume that $\theta=0$ and $r^{\prime} \geq 0$. Then, there exists at least a positive solution of (42) if

$$
\mu>\lambda_{1}\left(e^{\chi v_{r^{\prime}}} ; 1 ; N+r\right),
$$

and (42) does not possess a positive solution if

$$
0 \leq \mu \leq \lambda_{1}\left(1 ; e^{\chi v_{r^{\prime}}} ; N+r\right) .
$$

Corollary 5.2 Assume that $\theta=r^{\prime}=0$. Then, there exists at least a positive solution of (42) if, and only if,

$$
\mu>\lambda_{1}(1 ; 1 ; N+r)
$$

In order to prove the main result we are going to use a decoupling method and a subsupersolution method for non-local problems. Let us begin showing the validity of the this last method.

Consider a continuous map $B: \mathcal{C}(\bar{\Omega}) \mapsto \mathcal{C}(\bar{\Omega})$ and the non-linear equation

$$
\begin{cases}-\Delta u=f(x, u, B(u)) & \text { in } \Omega, \\ \frac{\partial u}{\partial n}+r(x) u=h(x) & \text { on } \partial \Omega,\end{cases}
$$

where $f: \Omega \times \mathbb{R} \times \mathcal{C}(\bar{\Omega}) \mapsto \mathbb{R}$ is a regular function; $r, h \in \mathcal{C}(\partial \Omega)$.

Definition 5.3 We say that $\underline{u}, \bar{u} \in \mathcal{C}^{2}(\Omega) \cap \mathcal{C}(\bar{\Omega})$ is a sub-supersolution of (46) if $\underline{u} \leq \bar{u}$ in $\Omega$ and

a)

$$
-\Delta \underline{u}-f(x, \underline{u}, B(u)) \leq 0 \leq-\Delta \bar{u}-f(x, \bar{u}, B(u)) \quad \text { in } \Omega, \forall u \in[\underline{u}, \bar{u}] \text {, }
$$

b)

$$
\frac{\partial \underline{u}}{\partial n}+r(x) \underline{u} \leq h(x) \leq \frac{\partial \bar{u}}{\partial n}+r(x) \bar{u} \quad \text { on } \partial \Omega .
$$


Proposition 5.4 Assume that there exists a sub-supersolution of (46), $\underline{u}, \bar{u}$, in the sense of Definition 5.3. Then, there exists a solution $u \in[\underline{u}, \bar{u}]$ of $(46)$.

Proof. Being the proof standard, we outline only it. Take $M>0$ large enough such that $u \mapsto f(x, u, \xi)+M u$ is increasing for all $x \in \Omega$ and $\xi \in \mathcal{C}(\bar{\Omega})$ and $\lambda_{1}(1 ; 1 ; N+r)+M>0$. Consider the map

$$
\begin{aligned}
\mathcal{T}:[\underline{u}, \bar{u}]_{2} & \rightarrow[\underline{u}, \bar{u}]_{2} \\
w & \mapsto u:=\mathcal{T}(w),
\end{aligned}
$$

being $u$ the unique solution of the following problem

$$
\left\{\begin{aligned}
-\Delta u+M u & =f(x, w, B(w))+M w & & \text { in } \Omega, \\
\frac{\partial u}{\partial n}+r(x) u & =h(x) & & \text { on } \partial \Omega,
\end{aligned}\right.
$$

where

$$
[\underline{u}, \bar{u}]_{2}:=\left\{u \in L^{2}(\Omega): \underline{u} \leq u \leq \bar{u}\right\} .
$$

It is not hard to show that we can apply the Schauder fixed point theorem to $\mathcal{T}$ and conclude the result.

To study system (42) we are going to apply the change of variable

$$
u=e^{\chi v} w
$$

which transforms the first equation of (42) into

$$
\begin{cases}-\operatorname{div}\left(e^{\chi v} \nabla w\right)=\mu e^{\chi v} w\left(1-e^{\chi v} w\right) & \text { in } \Omega \\ \frac{\partial w}{\partial n}+r w=r \theta e^{-\chi v} & \text { on } \partial \Omega .\end{cases}
$$

With respect to this equation, we get:

Proposition 5.5 Fix $v \in \mathcal{C}(\bar{\Omega})$ and denote by

$$
v_{L}:=\min _{x \in \bar{\Omega}} v(x), \quad v_{M}:=\max _{x \in \bar{\Omega}} v(x) .
$$

a) Assume that $r \theta>0$. Then, there exists a unique positive solution, denoted $w$, of (48) for $\mu \geq 0$. Moreover,

$$
\begin{array}{ll}
\min \{1, \theta\} e^{-\chi v_{M}} \leq w \leq \max \{1, \theta\} e^{-\chi v_{L}} & \text { if } \mu>0 \\
\theta e^{-\chi v_{M}} \leq w \leq \theta e^{-\chi v_{L}} & \text { if } \mu=0 .
\end{array}
$$

b) Assume that $r \theta=0$. Then, if $\mu>0$ there exists a unique positive solution of (48) if, and only if,

$$
\mu>\lambda_{1}\left(e^{\chi v} ; e^{\chi v} ; N+r\right) .
$$

Moreover,

$$
\frac{\mu-\lambda_{1}\left(e^{\chi v} ; e^{\chi v} ; N+r\right)}{\mu e^{\chi v_{M}}\|\varphi\|_{\infty}} \varphi \leq w \leq e^{-\chi v_{L}}
$$

where $\varphi$ is a positive eigenfunction associated to $\lambda_{1}\left(e^{\chi v} ; e^{\chi v}, N+r\right)$.

If $\mu=0$ there exists a positive solution if, and only if, $r=0$. In such case, any positive constant is solution. 
Proof. We will apply the sub-supersolution method.

a) Assume that $r \theta>0$. Take $\underline{w}:=\varepsilon>0$ and $\bar{w}:=K>0$ with $\varepsilon, K$ to be chosen. Then, $\varepsilon$ and $K$ must satisfy

$$
0 \leq \mu\left(1-e^{\chi v} \varepsilon\right) \quad \text { in } \Omega, \quad \varepsilon \leq \theta e^{-\chi v} \quad \text { on } \partial \Omega,
$$

and

$$
0 \geq \mu\left(1-e^{\chi v} K\right) \quad \text { in } \Omega, \quad K \geq \theta e^{-\chi v} \quad \text { on } \partial \Omega .
$$

This proves (49). The uniqueness follows because the map $w \mapsto \mu\left(1-e^{\chi v} w\right)$ is decreasing when $\mu>0$, see [5]. In the case $\mu=0$ the equation (48) is linear, and hence it is clear the uniqueness result.

b) Assume $r \theta=0$. Let $w$ be a positive solution of (48). Then,

$$
-\operatorname{div}\left(e^{\chi v} \nabla w\right)<\mu e^{\chi v} w \quad \text { in } \Omega, \quad \frac{\partial w}{\partial n}+r w=0 \quad \text { on } \partial \Omega,
$$

and so, multiplying by $\varphi$ and integrating we get that $\mu>\lambda_{1}\left(e^{\chi v} ; e^{\chi v} ; N+r\right)$. For the existence of solution take in this case $\underline{w}:=\varepsilon \varphi$ with

$$
\varepsilon=\frac{\mu-\lambda_{1}\left(e^{\chi v} ; e^{\chi v} ; N+r\right)}{\mu e^{\chi v_{M}}\|\varphi\|_{\infty}}
$$

Again, the uniqueness follows similarly.

Finally, the case $\mu=0$ follows easily.

We fix now $\mu>0$, the case $\mu=0$ will be treated separately. Fix $v \in \mathcal{C}(\bar{\Omega})$ and consider the equation (48). Denote by

$$
w(v):= \begin{cases}w_{v} & \text { if } r \theta>0 \text { or if } r \theta=0 \text { and } \mu>\lambda_{1}\left(e^{\chi v} ; e^{\chi v} ; N+r\right), \\ 0 & \text { in other case }\end{cases}
$$

being $w_{v}$ the unique positive solution of (46), which exists by Proposition 5.5.

Lemma 5.6 The operator $v \in \mathcal{C}(\bar{\Omega}) \mapsto w(v) \in \mathcal{C}(\bar{\Omega})$ is continuous.

Proof. Consider sequences $v_{n} \rightarrow v$ in $\mathcal{C}(\bar{\Omega})$ and $w_{n}:=w\left(v_{n}\right)$. Observe that $w_{n}$ is solution of

$$
-\operatorname{div}\left(a_{n}(x) \nabla w_{n}\right)+w_{n}=h_{n}, \quad \text { in } \Omega, \quad a_{n}(x) \frac{\partial w_{n}}{\partial n}=g_{n} \quad \text { on } \partial \Omega,
$$

being

$$
a_{n}(x):=e^{\chi v_{n}}, \quad h_{n}:=w_{n}+\mu w_{n} \cdot\left(1-e^{\chi v_{n}} w_{n}\right), \quad g_{n}:=a_{n} \cdot\left(-r w_{n}+r \theta e^{-\chi v_{n}}\right) .
$$

Observe that

$$
0<\alpha \leq a_{n} \leq \beta<\infty
$$

and thanks to (49) and (50) we get that $\left\|h_{n}\right\|_{\infty} \leq C$ and $\left\|g_{n}\right\|_{L^{\infty}(\partial \Omega)} \leq C$, and then, by Theorem 2.1 in [3], it follows that

$$
\left\|w_{n}\right\|_{\mathcal{C}^{\nu}(\bar{\Omega})} \leq C
$$


for $\nu \in(0,1)$ and some constants depending on $\alpha$ and $\beta$. Hence, for a subsequence, $w_{n} \rightarrow w$ in $\mathcal{C}(\bar{\Omega})$, being $w$ a weak solution of (46). The elliptic regularity proves that $w$ is a classical solution of (46). Now, we can follow the lines of the proof of Lemma 3.1 of [4] to conclude that in fact $w=w(v)$.

Proof of Theorem 5.1. Now, we have to study the following non-local and nonlinear equation

$$
\begin{cases}-\Delta v+v=\frac{e^{\chi v} w(v)}{1+e^{\chi v} w(v)} & \text { in } \Omega, \\ \frac{\partial v}{\partial n}+r^{\prime} v=r^{\prime} \frac{\theta}{2} & \text { on } \partial \Omega .\end{cases}
$$

To study this equation we apply Proposition 5.4 with $\bar{v}=v_{r^{\prime}}$ being $v_{r^{\prime}}$ the unique positive solution of (44).

We take as subsolution $\underline{v}=0$. Observe that $\underline{v}=0$ is sub-solution and no solution of (51) if

$$
\theta r^{\prime} \geq 0 \quad \text { and } \quad w(v) \geq 0 \quad \forall v \in\left[0, v_{r^{\prime}}\right]
$$

and some inequality strict. It is clear that this holds if $\theta r^{\prime}>0$ and paragraph a) follows for $\mu>0$.

Assume now that $r^{\prime}=0$ and $\theta>0$. If $r>0$ then $r \theta>0$ and by Proposition 5.5 we have that

$$
w(v) \geq \min \{1, \theta\} e^{-\chi v_{M}}>0 \text { for all } \mu>0 .
$$

If $r=0$, by Proposition 5.5

$$
\frac{\mu-\lambda_{1}\left(e^{\chi v} ; e^{\chi v} ; N+r\right)}{\mu e^{\chi v_{M}}\|\varphi\|_{\infty}} \varphi \leq w(v)
$$

if

$$
\mu>\lambda_{1}\left(e^{\chi v} ; e^{\chi v} ; N\right)=0
$$

Hence, $w(v)>0$ if $\mu>0$.

Finally assume that $r^{\prime} \geq 0$ and $\theta=0$. If $r=0$, by a similar reasoning, we need that $\mu>0$. If $r>0$ we need that

$$
\mu>\lambda_{1}\left(e^{\chi v} ; e^{\chi v} ; N+r\right) \quad \forall v \in\left[0, v_{r^{\prime}}\right] .
$$

Thanks to (45) we get

$$
\lambda_{1}\left(e^{\chi v} ; e^{\chi v} ; N+r\right) \leq \lambda_{1}\left(e^{\chi v_{r^{\prime}}} ; 1 ; N+r\right),
$$

hence it is enough that $\mu>\lambda_{1}\left(e^{\chi v_{r^{\prime}}} ; 1 ; N+r\right)$.

Finally, we show the non-existence result. Assume that $r \theta=0$. Observe that $w(v)=0$ if $\mu>0$ and $\mu \leq \lambda_{1}\left(e^{\chi v} ; e^{\chi v} ; N+r\right)$. But

$$
\lambda_{1}\left(e^{\chi v} ; e^{\chi v} ; N+r\right) \geq \lambda_{1}\left(1 ; e^{\chi v_{r^{\prime}}} ; N+r\right)
$$

whence we deduce the result.

Consider now the case $\mu=0$. If $r \theta>0$ then there exists a unique $w(v)>0$ solution of (48) and the result follows in a similar way. If $\theta=0$ and $r>0$ then $w(v)=0$, and then $u=0$. However, if $r=0$, we obtain that $w=C$ for any positive constant $C$, and then

$$
u=e^{\chi v} C .
$$


It is enough now to study the equation for $v$

$$
-\Delta v+v=\frac{C e^{\chi v}}{1+C e^{\chi v}} \quad \text { in } \Omega, \quad \frac{\partial v}{\partial n}+r^{\prime} v=r^{\prime} \frac{\theta}{2} \quad \text { on } \partial \Omega .
$$

We can argue as in the above case and conclude the result.

With respect to the semitrivial and trivial solutions, we get:

Proposition $\mathbf{5 . 7} \quad$ a) The trivial solution $(u, v)=(0,0)$ exists if and only if $r \theta=r^{\prime} \theta=$ 0 . In such case, the solution is unstable for $\mu>\lambda_{1}(1 ; 1 ; N+r)$ and stable for $\mu<\lambda_{1}(1 ; 1 ; N+r)$.

b) The semitrivial solution $\left(0, V_{r^{\prime}}\right)$ exists if and only if $r=0$ and $r^{\prime}, \theta>0$, being $V_{r^{\prime}}$ the unique solution of

$$
\begin{cases}-\Delta V+V=0 & \text { in } \Omega, \\ \frac{\partial V}{\partial n}+r^{\prime} V=r^{\prime} \frac{\theta}{2} & \text { on } \partial \Omega .\end{cases}
$$

In such case, $\left(0, w_{r^{\prime}}\right)$ is unstable for $\mu>0$.

Proof. a) The existence result is not hard to show. On the other hand, the stability of $(0,0)$ is given by the real parts of the eigenvalues for which the following problem admits a solution $(\xi, \eta) \neq(0,0)$

$$
\begin{cases}-\Delta \xi-\mu \xi=\sigma \xi & \text { in } \Omega, \\ -\Delta \eta+\eta=\xi+\sigma \eta & \text { in } \Omega, \\ \frac{\partial \xi}{\partial n}+r \xi=0 & \text { on } \partial \Omega, \\ \frac{\partial \eta}{\partial n}+r^{\prime} \eta=0 & \text { on } \partial \Omega .\end{cases}
$$

If $\xi=0$, then

$$
\sigma=\lambda_{j}\left(1 ; 1 ; N+r^{\prime}\right)+1>0 .
$$

Assume now that $\xi \neq 0$, so

$$
\sigma=\lambda_{j}(1 ; 1 ; N+r)-\mu \geq \lambda_{1}(1 ; 1 ; N+r)-\mu .
$$

Then, if $\mu<\lambda_{1}(1 ; 1 ; N+r)$ we obtain that $\sigma>0$ and $(0,0)$ is stable.

Assume now that $\mu>\lambda_{1}(1 ; 1 ; N+r)$. Then,

$$
\sigma_{1}:=\lambda_{1}(1 ; 1 ; N+r)-\mu<0 .
$$

Denote by $\xi$ a positive eigenfunction associated to $\sigma_{1}$, that is

$$
-\Delta \xi-\mu \xi=\sigma_{1} \xi \quad \text { in } \Omega, \quad \frac{\partial \xi}{\partial n}+r \xi=0 \quad \text { on } \partial \Omega .
$$

Since $\sigma_{1}<0$, then

$$
\lambda_{1}\left(1 ; 1 ; N+r^{\prime}\right)+1-\sigma_{1}>0,
$$

and so there exists $\eta \neq 0$ such that

$$
-\Delta \eta+\eta-\sigma_{1} \eta=\xi \quad \text { in } \Omega, \quad \frac{\partial \eta}{\partial n}+r^{\prime} \eta=0 \quad \text { on } \partial \Omega .
$$


Then, $\sigma_{1}<0$ is an eigenvalue of (53) with associated eigenfunction $(\xi, \eta)$, so $(0,0)$ is unstable.

b) Again the existence result is direct. Now, the linearization around $\left(0, V_{r^{\prime}}\right)$ is

$$
\begin{cases}-\Delta \xi=-\operatorname{div}\left(\chi \xi \nabla V_{r^{\prime}}\right)+\mu \xi+\sigma \xi & \text { in } \Omega, \\ -\Delta \eta+\eta=\xi+\sigma \eta & \text { in } \Omega, \\ \frac{\partial \xi}{\partial n}-\chi \xi \frac{\partial V_{r^{\prime}}}{\partial n}=0 & \text { on } \partial \Omega, \\ \frac{\partial \eta}{\partial n}+r^{\prime} \eta=0 & \text { on } \partial \Omega .\end{cases}
$$

The first equation, after the change of variable

$$
\xi=e^{\chi V_{r^{\prime}}} \psi
$$

is transformed into

$$
-\operatorname{div}\left(e^{\chi V_{r^{\prime}}} \nabla \psi\right)=(\mu+\sigma) e^{\chi V_{r^{\prime}}} \psi \quad \text { in } \Omega, \quad \frac{\partial \psi}{\partial n}=0 \quad \text { on } \partial \Omega .
$$

If $\xi=0$ then, since $\eta \neq 0$, then $\sigma=\lambda_{j}\left(1 ; 1 ; N+r^{\prime}\right)+1>0$. On the other hand, if $\xi \neq 0$ we get

$$
\sigma=\lambda_{j}\left(e^{\chi V_{r^{\prime}}} ; e^{\chi V_{r^{\prime}}} ; N\right)-\mu \geq \lambda_{1}\left(e^{\chi V_{r^{\prime}}} ; e^{\chi V_{r^{\prime}}} ; N\right)-\mu>0,
$$

since $\lambda_{1}\left(e^{\chi V_{r^{\prime}}} ; e^{\chi V_{r^{\prime}}} ; N\right)=0$ and $\mu<0$.

Assume now that $\mu>0$, then $\sigma_{1}:=\lambda_{1}\left(e^{\chi V_{r^{\prime}}} ; e^{\chi V_{r^{\prime}}} ; N\right)-\mu<0$ and consider $\psi$ a positive eigenfunction associated to $\sigma_{1}$, that is

$$
-\operatorname{div}\left(e^{\chi V_{r^{\prime}}} \nabla \psi\right)=\left(\mu+\sigma_{1}\right) e^{\chi V_{r^{\prime}}} \psi \quad \text { in } \Omega, \quad \frac{\partial \psi}{\partial n}=0 \quad \text { on } \partial \Omega .
$$

Again, consider the change of variable $\xi=e^{\chi V_{r^{\prime}}} \psi$, and $\eta$ the solution of

$$
-\Delta \eta+\eta=\xi+\sigma_{1} \eta \quad \text { in } \Omega, \quad \frac{\partial \eta}{\partial n}+r^{\prime} \eta=0 \quad \text { on } \partial \Omega,
$$

which exists because $\lambda_{1}\left(1 ; 1 ; N+r^{\prime}\right)+1-\sigma_{1}>0$. Then, $\sigma_{1}<0$ is an eigenvalue of (54) with associated eigenfunction $(\xi, \eta)$, so $\left(0, V_{r^{\prime}}\right)$ is unstable.

\section{References}

[1] S. Agmon, A. Douglis and L. Nirenberg, Estimates near the boundary for solutions of elliptic partial differential equations satisfying general boundary conditions. Comm. Pure Appl. Math. 12, (1959) 623-727.

[2] H. Amann, Nonhomogeneous Linear and Quasilinear Elliptic and Parabolic Boundary Value Problems, Eds. Schmeisser and Triebel, Function Spaces, Differential Operators and Nonlinear Analysis, Teubner Texte zur Mathematik, 133 (1993) 9-126.

[3] D. Arcoya and J. D. Rossi, Antimaximum principle for quasilinear problems, Adv. Differential Equations 9 (2004), 1185-1200. 
[4] J. Blat and K. J. Brown, Bifurcation of steady-state solutions in predator-prey and competition systems, Proc. Roy. Soc. Edinburgh Sect. A 97 (1984), 21-34.

[5] H. Brezis and L. Oswald, Remarks on sublinear elliptic equations, Nonlinear Anal., 10 (1986) 55-64.

[6] R. S. Cantrell, C. Cosner and S. Martínez, Global bifurcation of solutions to diffusive logistic equations on bounded domains subject to nonlinear boundary conditions, Proc. Roy. Soc. Edinburgh Sect. A 139 (2009), 45-56.

[7] M. A. J. Chaplain and G. Lolas, Mathematical modelling of cancer cell invasion of tissue: the role of the urokinase plasminogen activation system, Math. Models Methods Appl. Sci. 15 (2005), 1685-1734.

[8] M. Delgado, I. Gayte, C. Morales-Rodrigo and A. Suárez, An angiogenesis model with nonlinear chemotactic response and flux at the tumor boundary, Nonlinear Analysis (2009), doi:10.1016/j.na.2009.06.057

[9] D. Henry, Geometric theory of semilinear parabolic equations, Lecture Notes Math. 840, Springer 1981.

[10] M. R. Myerscough, P. K. Maini and K. J. Painter, Pattern formation in a generalized chemotactic model, Bulletin of Mathematical Biology 60 (1998), 1-26.

[11] J. I. Tello and M. Winkler, On a Chemotaxis with logistic source, Communications in Partial Differential Equations, 32 (2007), 849 - 877.

[12] M. J. Tindall, P. K. Maini, S. L. Porter and J. P. Armitage, Overview of mathematical approaches used to model bacterial chemotaxis. II. Bacterial populations. Bull. Math. Biol. 70 (2008), 1570-1607

[13] M. Winkler, Chemotaxis with logistic source: very weak global solutions and their boundedness properties, J. Math. Anal. Appl. 348 (2008), 708-729. 